\title{
The Discrete-Time Sliding Mode Control with Computation Time Delay for Repeatable Run-Out Compensation of Hard Disk Drives
}

\author{
T. H. Yan, ${ }^{1}$ B. He, ${ }^{2}$ X. D. Chen, ${ }^{3}$ and X. S. Xu ${ }^{1}$ \\ ${ }^{1}$ College of Mechanical and Electrical Engineering, China Jiliang University, 528 Xueyuan Road, \\ Xiasha Univ Park, Hangzhou 310018, China \\ ${ }^{2}$ School of Information Science and Engineering, Ocean University of China, Qingdao 266100, China \\ ${ }^{3}$ State Key Laboratory of Digital Manufacturing Equipment and Technology, Huazhong University of Science and Technology, \\ Wuhan 430074, China \\ Correspondence should be addressed to T. H. Yan; thyan@163.com and B. He; bhe@ouc.edu.cn
}

Received 22 August 2012; Accepted 9 January 2013

Academic Editor: Kwok-Wo Wong

Copyright (C) 2013 T. H. Yan et al. This is an open access article distributed under the Creative Commons Attribution License, which permits unrestricted use, distribution, and reproduction in any medium, provided the original work is properly cited.

\begin{abstract}
The presented is a study on the problem of disturbance rejection, specifically the periodic disturbance, by applying discretetime sliding mode control method. For perturbations such as modeling errors and external disturbances, their compensation is formulated using the designed sliding mode control. To eliminate the effect of these perturbations, the convergence rate between the disturbance and their compensation has been shaped by an additional parameter. Decoupling of the resultant perturbation estimation dynamics from the closed loop dynamics is achieved. Computation time delay is also presented to address the perturbation effects. The approach developed ensures the robustness of the sliding mode dynamics to parameter uncertainties and exogenous disturbances, in addition to the complete rejection of the periodic disturbance component. Satisfactory simulation results as well as experimental ones have been achieved based on a fast servo system of a modern hard disk drive to illustrate the validity of the controller for repeatable run-out (RRO) compensation.
\end{abstract}

\section{Introduction}

One of the most attractive features of the variable structure system (VSS) with sliding mode is its invariance and robustness to perturbations including modeling errors and external disturbances. Originated in the late 1950s, sliding mode theory has been developed mostly in the continuous time domain especially after the earliest works published by Itkis [1] and Utkin [2]. Thereafter, researches on this topic had increased rapidly, for example, Hui and Żak studied the discrete-time variable structure sliding mode control [3]; Kalsi et al. presented a high gain approach [4]. Employing describing function techniques, sliding mode control of DC servo mechanisms is analyzed in the presence of unmodeled stator and sensor dynamics by Xu et al. [5]. A sliding-modebased learning controller for track-following in hard disk drives was presented by Wu and Liu [6], and the computation time delay is treated as a fault to be detected, using an appropriate controller to minimize its effects by Garcia et al. [7].

Sliding mode requires high-speed discontinuous action to steer the states of a system into a sliding surface and to maintain the subsequent motion on that surface. Nowadays, digital computers have been widely used in all kinds of control systems. However, limited sampling frequency results in control inputs to be constant between two sampling intervals, leading to difficulties for instantaneous actions to be implemented in the sampled-data system. This means that when system dynamics cross the sliding surface between sampling intervals, the control input cannot immediately take measures to enable the system to remain on that sliding surface, leading to VSS controllers implemented in discrete-time system not to possess those desirable properties due to finite sampling time [8]. Therefore, implementation of continuous 
VSS controllers on digital computers presents difficulties due to limited sampling rate, sample/hold effects, and discretization errors which can possibly lead to unacceptable results [9].

In order to achieve the robustness required, much research on discrete-time variable structure control (DVSC) and discrete-time sliding mode control (DSMC) has been presented recently. Sarpturk et al. [10] and Kotta [11] showed the necessary but not sufficient conditions for achieving a sliding motion of discrete variable structure systems, and proposed a new and more strict condition required to be met. Hung et al. [12] proposed a reaching law for discretetime variable structure control. Gao et al. [13] formulated the concept of discrete sliding mode by making use of the reaching law proposed in [12] to ensure reaching conditions that are satisfied for stability and convergence of $s(k)$. Bartolini et al. [14] incorporated adaptive control strategy into the modeling of system uncertainties and designed a control law in terms of a discrete-time equivalent control. Tesfaye and Tomizuka [15] proposed the concept of time-delay control (TDC) and a robust discrete-time sliding mode control using the delta $(\delta)$ operator. The behavior of the discrete-time sliding mode controller for linear, nonlinear, and stochastic systems was investigated by $\mathrm{Su}$ et al. [16] who conducted a detailed analysis of sampling and hold effects. Koshkouei and Zinober [17] introduced the notation of the sliding lattice and clarified the concept of discrete-time sliding mode control (DSMC). Corradini and Orlando [18] employed the concept of TDC to eliminate the effects of system perturbation within switching region. With proper sampling period $T_{s}$, Su et al. [19] verified that the thickness of the boundary layer can be reduced to $O\left(T_{s}^{2}\right)$ for continuous smooth disturbance. Eun et al. [20] proposed a decoupled disturbance compensator (DDC) by directly using the variable structure framework and combined it with DVSC. In his DVSC with DDC structure, however, system response may become slow, and controller implementation can become rather complex. To enable tracking error vector to remain close to zero without using any additional disturbance estimation scheme, Kim and Cho [21] proposed a DVSC method with a recursive switching function (R-DVSC). After that, the R-DSVC with an additional parameter to tune the transient response was proposed by Furuta [9]. Furthermore, a sliding surface that allows the transient response to be shaped by introducing information of past states in the sliding surface was proposed by S. M. Lee and B. H. Lee [22].

Beside the limited sampling rate, there is an inherent computation time delay because of the existence of measurement delays of feedback signals as well as the execution time of instructions when a control algorithm is implemented on a digital computer. S. M. Lee and B. H. Lee [22] summarized some of the previous works. Kondo and Furuta [23] examined the computation time delay for an optimal full state-feedback regulator problem. Ha and Ly [24] formulated the computation time delay in $W$-domain. Misawa [25] showed that the presence of computation time delay not only reduces relative stability and robustness but also degrades performance and proposed that a controller that embraces the concept of TDC proposed by Tesfaye and Tomizuka [15] to compensate the effects of perturbations should be used. For the repetitive control of discrete-time systems, one of the earlier classical works was presented by Tomizuka et al. [26]. Recently, the discrete-time sliding-mode congestion control with timevarying delay for multisource communication networks was presented by Ignaciuk and Bartoszewicz [27]. Sliding mode control for time-varying delayed systems based on a reducedorder observer is considered by Yan et al. [28]. Due to its importance and wide use, a lot of scientists focused on high performance controller for real systems, for example, [2933]. Here, the high performance servo system for HDDs is investigated via discrete-time sliding mode approach.

Since discrete-time sliding mode control has been developed for guaranteeing the asymptotic stability of uncertain sampled-data systems, as well as to reducing the chattering phenomenon that arises when the controller was implemented on a digital computer, the computation time delay should also be considered in the discrete-time sliding mode structure for improving the system performance. This paper presents a discrete-time sliding mode controller with an additional parameter to adjust the convergence rate for improving the perturbation compensation, and then based on the spirit of [22], the effects of computation time delay are considered for complete disturbances compensation. Experiments have been implemented based on a modern hard disk drive (HDD). It is assumed that the computation time delay is constant and smaller than the sampling time. For the clarity of presentation, a controller with an additional adjustable parameter that does not take computation time delay into account is firstly presented. Then the controller that considers computation time delay is developed. By introducing an additional parameter, the difference between perturbation and its compensation convergences to zero asymptotically. The controller design law makes use of the concept of TDC to compensate the effects of perturbations. In the presence of the influences of perturbations by unknown external disturbances and parametric uncertainties, the developed controller generates a compensation signal to cancel these influences through the mechanism of time delay. The DSMC with decoupled estimator presented in this paper are intrinsic robust to parameter uncertainties and exogenous disturbances and especially effective with the periodical disturbances. For the real servo systems, for example, the hard disk drives have strong repeatable runouts (RROs) due to the eccentric characteristics of the rotating disk and shaft; the high-speed and high-precision multiaxis stages in wire bonder or the ICs (integrated circuits) exposure lithography equipments have the strong periodic disturbances during stepping under the operating modes. The periods of the disturbances are different when the operation modes are changed, and these could be estimated within control algorithm automatically. The positioning accuracy of the control systems could be improved significantly when taking the DSMC with the decoupled estimator when considering computational time delay.

The remains of the paper are organized as follows. Discrete-time models with and without computation time delay are briefly outlined in Section 2. In Section 3, a discrete-time-sliding mode controller with shaped perturbation 
rejection that does not consider the computation time delay is presented. Afterwards, the controller that accounts for the effects of computation time delay is developed. In Section 4, numerical simulations to verify the proposed control methods are conducted based on the servo-control model of a commercial HDD. In Section 5, experimental digital servo system for track-to-track seeking of HDD is implemented, from which the experimental measured results are provided to show the validity of the proposed method. Finally, conclusions are drawn in Section 6.

\section{Preliminaries}

Consider the following SISO LTI system with parametric uncertainties and exogenous disturbances described by the continuous-time model:

$$
\begin{gathered}
\dot{x}(t)=(F+\Delta F) x(t)+G u(t)+E \xi(t), \\
y(t)=H x(t),
\end{gathered}
$$

where $x$ is the $n$-dimensional state vector, $u \in R$ is the system input, $y \in R$ is the measured output, and $\xi \in R$ is the perturbation, which represents the effects of external disturbances and parametric uncertainties. $F, \Delta F, G, E$, and $H$ are properly dimensioned constant matrices. The continuoustime matching condition is assumed to hold; that is, there exists a $1 \times n$ row scalar vector $\Delta f$ and a scalar $\Delta_{\xi}$ such that $\Delta F=G \Delta f$ and $E=G \Delta_{\xi}$.

2.1. Discrete-Time Model without Computation Time Delay. The discrete-time model with sampling period $T_{s}$ is

$$
x(k+1)=A_{d} x(k)+b_{d} u(k)+D \xi(k),
$$

denoting with the index $k$ the variable evaluated in $t=k T_{s}$. In the above system, the pair $\left(A_{d}, b_{d}\right)$ is assumed to be controllable, and as is shown by Tesfaye and Tomizuka [15], the matching condition can be enforced in discrete system model, if a suitable sampling time is chosen by means of the assumption below.

Assumption 1 (see [15]). Let the sampling interval $T_{s}$ be small enough so that the first two terms in the Taylor expansion of a function give an acceptably close approximation. [18]:

Hence, the following discrete-time model can be obtained

$$
x(k+1)=A x(k)+b u(k)+b d(k),
$$

where $A, b, g$, and $d(k)$ are obtained as follows:

$$
\begin{gathered}
A=e^{F T_{s}}, \quad B=\int_{0}^{T_{s}} e^{F \tau} d \tau G, \\
\Delta A=\Delta f\left[I+(F+G \Delta f) \frac{T_{s}}{2}\right], \\
d(k)=\frac{\Delta A x(k)}{g}+\Delta_{\xi} \xi(k), \\
g=1+\Delta f G \frac{T_{s}}{2}, \quad b=B g .
\end{gathered}
$$

In (4), the disturbance is constructed as $d(k)=\Delta A x(k) /$ $g+\Delta_{\xi} \xi(k)$ including the parametric uncertainty $\Delta f$ and external disturbance vector $D \xi(k)$.

Assumption 2. Both the matching condition and the $(A, b)$ pair are completely controllable and are assumed to hold through the paper.

So, the switching function is defined as follows:

$$
s(k)=c^{T} x(k)=0 .
$$

2.2. Discrete-Time Model with Computation Time Delay. Assume there exists a delay $\delta$, which is caused mainly by the execution time of the instructions that generate the control input after the sampling instant when a control algorithm was implemented on a digital computer. Following S. M. Lee and B. H. Lee [22], it is assumed here that the delay is constant and smaller than one sampling interval $T_{s}\left(0<\delta<T_{s}\right)$, and this condition is assumed to hold through the paper. Therefore, the control input $u(t)$ should be chosen as follows:

$$
u(t)=u(k), \quad \text { for } k T_{s}+\delta \leq t<(k+1) T_{s}+\delta .
$$

The discrete-time model of (1) with control input (12) is given as follows $[22,23,34]$

$$
\begin{aligned}
x(k+1) & =A x(k)+b_{1} u(k-1)+b_{2} u(k)+D \xi(k) \\
& =A x(k)+b_{1} u(k-1)+b_{2} u(k)+b d(k),
\end{aligned}
$$

where $A=e^{F T_{s}}, b_{1}=\int_{T_{s}-\delta}^{T_{s}} e^{F \tau} d \tau G=b-b_{T_{s}-\delta}, b_{2}=b_{T_{s}-\delta}=$ $\int_{0}^{T_{s}-\delta} e^{F \tau} d \tau G$, and $b=\int_{0}^{T_{s}} e^{F \tau} d \tau G$. In spite of the existence of computation time delay, the controllability and observability assumptions are preserved in this model. Although the discrete-time matching condition is not satisfied in system (8), the effects of perturbation can be sufficiently suppressed under the assumption that the perturbation is relatively slower than the sampling frequency $1 / T_{s}$.

\section{Discrete-Time Sliding Mode Control Design}

The concept of TDC [15] consists of estimating the uncertain dynamics of the system through past observations of the system response, so that a control function can be generated (with some delay) to approximately counterbalance their effects. In order to cancel the effects of perturbation inside the switching region, Corradini and Orlando [18] proposed a discrete-time VSC, in which the perturbation estimator was formulated by using the concept of TDC. Furthermore, a sliding surface was selected to allow the transient time response to be shaped by introducing the past states in the sliding surface. In discrete-time systems, instead of having a hyperplane as in the case of continuous time, a countable set of points is defined a comprising the so-called lattice, and the surface on which these sliding points lie is named the latticewise hyperplane $[17,35]$. Here, using the concept of discretetime sliding mode illustrated by the sliding lattice, the DSMC with a decoupled perturbation compensator (DPC) that does not consider computation time delay is firstly presented. Then 
regarding the inherent computation time delay, an improved DSMC with DPC is presented.

3.1. Discrete-Time Sliding Mode Control Design without Computation Time Delay. Considering the discrete-time linear time-invariant system given by (10), the discrete-time equivalent control for the nominal plant without perturbation can be obtained by solving the equation $s(k+1)=0$ as

$$
u_{\mathrm{eq}}(k)=-\left(c^{T} b\right)^{-1} c^{T} A x(k)
$$

The control objective of the discrete-time sliding mode control is to achieve $s(k+1)=s(k)=0$. When $s(k)=0$, that is, when the state vector remains on the sliding surface, the closed-loop dynamics becomes

$$
x(k+1)=\left[I-b\left(c^{T} b\right)^{-1} c^{T}\right] A x(k) .
$$

The state vector $x(k)$ remains on the sliding surface defined in (11) and converges to the origin in the state space, if $\left[I-b\left(c^{T} b\right)^{-1} c^{T}\right]$ is a contractive matrix.

Based on the spirit of the reaching law proposed by Gao et al. [13], the transient response can be shaped by introducing an additional adjustable parameter to the desired sliding function dynamics such that $s(k+1)=\beta s(k)$. By solving this equation, the control input that can shape the transient response is obtained as follows:

$$
u_{\beta}(k)=-\left(c^{T} b\right)^{-1} c^{T} A x(k)+\left(c^{T} b\right)^{-1} \beta s(k), \quad 0 \leq \beta<1 .
$$

This control law for the nominal plant can be modified for the system with the unknown perturbations. If $v(k)$ denotes the perturbation compensation input, the following modified control input is obtained:

$$
u(k)=u_{\beta}(k)+v(k) .
$$

Then, substituting (11) into the expression of $s(k+1)$ defined by (6), the following equation can be obtained:

$$
s(k+1)=\left(c^{T} b\right)[v(k)+d(k)]+\beta s(k) .
$$

From (13), we have

$$
v(k)=-d(k)+\left(c^{T} b\right)^{-1}[s(k+1)-\beta s(k)] .
$$

The additive input term $v(k)$ ensures the stability and robustness of the closed-loop system under the presence of perturbations [18], [36]. However, the control cannot be actually implemented because of a lack of knowledge about $d(k)$. If $\xi(t)$ is bounded and considerably slower than the sampling frequency $1 / T_{s}$, the difference between $d(k)$ and $d(k-1)$ is of $O\left(T_{s}\right)$ [36]. If the disturbance is smooth function, the difference between $d(k)$ and $d(k-1)$ is of $O\left(T_{s}^{2}\right)$ [16], so $d(k-1)$ can be used as an estimate of $d(k)$. From (14), a onestep backward shifted perturbation becomes

$$
d(k-1)=-v(k-1)+\left(c^{T} b\right)^{-1}[s(k)-\beta s(k-1)] .
$$

The compensation input $v(k)$ is defined as an estimate of $-d(k): v(k)=-\widehat{d}(k)=-d(k-1)$. So from (15), the perturbation compensation law can be obtained as

$$
v(k)=v(k-1)-\left(c^{T} b\right)^{-1}[s(k)-\beta s(k-1)] .
$$

Obviously, (16) is the separate disturbance estimator, and the convergence rate of $|d(k)-v(k)|$ is only related to $\left(c^{T} b\right)^{-1}[s(k)-\beta s(k-1)]$. If the perturbation is constant or slowly varying, an adjustable parameter $\alpha(0<\alpha \leq 1)$ is added to the item that contains the switching function in (16) to make $|d(k)-v(k)|$ converges asymptotically to zero in a desired sliding function dynamics, leading to the following equations to be obtained:

$$
\begin{aligned}
& d(k-1)=-v(k-1)+\alpha\left(c^{T} b\right)^{-1}[s(k)-\beta s(k-1)], \\
& v(k)=v(k-1)-\alpha\left(c^{T} b\right)^{-1}[s(k)-\beta s(k-1)] .
\end{aligned}
$$

Equation (18) is the separate disturbance estimator to be used to compensate system perturbations. The transient response has been shaped by introducing the past state in sliding surface. It can be easily shown that this control law forces the system to be reached at the discrete-time sliding mode and the perturbation can be compensated in a finite time instant, if $|d(k)-d(k-1)|$ is a decreasing sequence for $k>1$ $[37,38]$.

Theorem 1. For the system described in (4), if one chooses the control law (11) and (12) and the disturbance compensation law (18), with $\varepsilon(k)=d(k)+v(k)$ being the compensation error, the following closed-loop sliding mode dynamics and compensation error dynamics are satisfied:

$$
\begin{gathered}
s(k+1)=\left(c^{T} b\right) \varepsilon(k)+\beta s(k), \\
\varepsilon(k+1)=(1-\alpha) \varepsilon(k)+d(k+1)-d(k) .
\end{gathered}
$$

Proof. From (13), (14) can be easily proven.

By substituting (16) and (19) into the equation $\varepsilon(k)=$ $d(k)+v(k),(20)$ can be derived as follows:

$$
\begin{aligned}
\varepsilon(k+1) & =d(k+1)+v(k+1) \\
& =d(k+1)+v(k)-\alpha\left(c^{T} b\right)^{-1}[s(k+1)-\beta s(k)] \\
& =d(k+1)-d(k)+(1-\alpha) \varepsilon(k) .
\end{aligned}
$$

Theorem 1 implies that the disturbance estimation dynamics $\varepsilon(k)$ and the sliding mode dynamics $s(k)$ are decoupled. It is similar to the separation principle, allowing both dynamics modes to be tuned separately. The developments for the robustness of the proposed method are presented in the following theorems. 
Theorem 2. If $|d(k)-d(k-1)|<\xi$ for (20) holds for with $0<$ $\alpha \leq 1$, and for some constant $\xi>0$, then for all $k>k^{\prime}$, there exists some integer $k^{\prime}$ such that

$$
|\varepsilon(k+1)|<\frac{\xi}{\alpha} .
$$

Proof. It follows from (20) that

$$
\begin{aligned}
|\varepsilon(k+1)| & =|(1-\alpha) \varepsilon(k)+d(k+1)-d(k)| \\
& \leq(1-\alpha)|\varepsilon(k)|+|d(k+1)-d(k)| .
\end{aligned}
$$

Since $|d(k)-d(k-1)|<\xi$, for all $k$, the compensation error satisfies

$$
|\varepsilon(k+1)|<(1-\alpha)|\varepsilon(k)|+\xi .
$$

Iterating the above inequality gives rise to

$$
\begin{aligned}
|\varepsilon(k+1)| & \\
& <(1-\alpha)|\varepsilon(k)|+\xi \\
& \leq(1-\alpha)^{2}|\varepsilon(k-1)|+(1-\alpha) \xi+\xi \\
& \vdots \\
& \leq(1-\alpha)^{r}|\varepsilon(k-r+1)|+\sum_{i=1}^{r}(1-\alpha)^{r-i} \xi .
\end{aligned}
$$

This implies

$$
\begin{aligned}
|\varepsilon(k+1)| \leq & (1-\alpha)^{r} \max \{|\varepsilon(1)|,|\varepsilon(2)|, \ldots,|\varepsilon(r)|\} \\
& +\frac{\left[1-(1-\alpha)^{r-i}\right] \xi}{\alpha} \\
& \text { and as } k \longrightarrow \infty, \quad \lim _{k \rightarrow \infty} \sup |\varepsilon(k+1)| \leq \frac{\xi}{\alpha} .
\end{aligned}
$$

It is shown in Theorem 2 that the compensation error in (20) converges asymptotically to zero if the disturbance is constant or of slowly varying nature.

Theorem 3. If $|d(k)-d(k-1)|<\xi$ with $0<\alpha \leq 1$ and $0 \leq \beta<$ 1 for (19) and (20), for all $k$ and $\xi>0$, then there exists some integer $k_{0}$ such that for all $k>k_{0}$ we have

$$
|s(k+1)| \leq \frac{\left|\left(c^{T} b\right)\right| \xi}{[\alpha(1-\beta)]}
$$

Proof. It follows from (20) that

$$
\begin{aligned}
\mid s(k+ & 1) \mid \\
& =\left|\left(c^{T} b\right) \varepsilon(k)+\beta s(k)\right| \\
& \leq\left|\left(c^{T} b\right) \varepsilon(k)\right|+\beta|s(k)| \\
& \leq\left|\left(c^{T} b\right)\right| \frac{\xi}{\alpha}+\beta|s(k)| \\
& \leq\left|\left(c^{T} b\right)\right| \frac{\xi}{\alpha}+\beta^{2}|s(k-1)|+\frac{\beta\left|\left(c^{T} b\right)\right| \xi}{\alpha} \\
& \vdots \\
& \leq \beta^{k-k_{0}}\left|s\left(k_{0}\right)\right|+\frac{\sum_{i=1}^{k-k_{0}} \beta^{k-k_{0}-i}\left|\left(c^{T} b\right)\right| \xi}{\alpha} \\
& =\beta^{k-k_{0}}\left|s\left(k_{0}\right)\right|+\frac{(1-\beta)^{k-k_{0}}\left|\left(c^{T} b\right)\right| \xi}{[\alpha(1-\beta)]} \\
& \text { and as } k \longrightarrow \infty, \lim _{k \rightarrow \infty} \sup |s(k+1)| \leq \frac{\left|\left(c^{T} b\right)\right| \xi}{[(1-\beta) \alpha]}
\end{aligned}
$$

The robustness of the closed-loop sliding mode dynamics to the disturbance is guaranteed from Theorem 3 and the asymptotic bound on the switching function is proportional to the bound on $|d(k)-d(k-1)|$. By Theorem 3, the switching function is ensured to converge to a bound proportional to the reciprocal of adjustable parameters $\alpha$ and $1-\beta$.

Similarly, the theorems can be proposed and proved for the periodic perturbation compensation. To the periodic disturbance with known period $N$, and if $N$ is assumed slower than the sampling frequency $1 / T_{s}$, then the compensation input $v(k)$ is defined as an estimate of $-d(k-N)$. From (18), the compensation for periodic disturbance is obtained as

$$
v(k)=v(k-N)-\alpha\left(c^{T} b\right)^{-1}[s(k-N+1)-\beta s(k-N)] .
$$

It can be easily shown that this control law forces the system to be reached at the discrete-time sliding mode and the periodic disturbance can be compensated in a finite time instant, if $|d(k)-d(k-N)|$ is a decreasing sequence for $k>1$ $[37,39]$.

3.2. Discrete-Time Sliding Mode Control Design with Computation Time Delay. The discrete-time sliding mode controller considering the effects of computation time delay is considered here. Similar to the control law mentioned in the previous section, the new control law utilizes the concept of TDC to formulate the perturbation estimation process [36]. 
Considering the discrete-time model with computation time delay described by (13), the following equivalent control can be obtained for the nominal plant without perturbation:

$$
u_{\mathrm{eq}}(k)=-\left(c^{T} b_{2}\right)^{-1} c^{T} A x(k)-\left(c^{T} b_{2}\right)^{-1}\left(c^{T} b_{1}\right) u(k-1) .
$$

For the desired sliding function dynamics $s(k+1)=\beta s(k)$, the control input that can shape the transient response is obtained as

$$
u_{\beta}(k)=u_{\mathrm{eq}}(k)+\left(c^{T} b_{2}\right)^{-1} \beta s(k), \quad(0 \leq \beta<1) .
$$

For the compensation of unknown perturbation, the perturbation compensation input $v(k)$ is added to the above control input, leading to a control law as

$$
u(k)=u_{\beta}(k)+v(k) \text {. }
$$

Note that the control input $u(k-1)$ is included in the equivalent control input $u_{\mathrm{eq}}(k)$. Since $u(k-1)$ contains the time delay control input $v(k-1)$, the input $u_{\mathrm{eq}}(k)$ that is defined for the nominal plant should be modified to exclude the control term $v(k-1)$. From (32), we have $u(k-1)=$ $u_{\beta}(k-1)+v(k-1)$ and redefine the equivalent control input $u_{\text {eq }}(k)$ for nominal system as follows:

$$
u_{\mathrm{eq}}(k)=-\left(c^{T} b_{2}\right)^{-1} c^{T} A x(k)-\left(c^{T} b_{2}\right)^{-1}\left(c^{T} b_{1}\right) u_{\beta}(k-1) .
$$

So, the control law with compensation for the unknown perturbation can be obtained as

$$
\begin{aligned}
u(k)= & -\left(c^{T} b_{2}\right)^{-1} c^{T} A x(k)-\left(c^{T} b_{2}\right)^{-1}\left(c^{T} b_{1}\right) u_{\beta}(k-1) \\
& +\left(c^{T} b_{2}\right)^{-1} \beta s(k)+v(k) .
\end{aligned}
$$

In fact, the control cannot be actually implemented because of a lack of knowledge of current $d(k)$. Under the assumption that the perturbation is bounded and much slower than the sampling frequency $1 / T_{s}, d(k-1)$ can be used as an estimate of $d(k)$. Substituting (34) into the expression of $s(k+1)=0$, the following equation is obtained:

$$
\begin{aligned}
d(k)= & -\left(c^{T} b\right)^{-1}\left[\left(c^{T} b_{2}\right) v(k)+\left(c^{T} b_{1}\right) v(k-1)\right] \\
& +\left(c^{T} b\right)^{-1}[s(k+1)-\beta s(k)] .
\end{aligned}
$$

Based on (35), a one-step backward shifted perturbation becomes

$$
\begin{aligned}
d(k-1)= & -\left(c^{T} b\right)^{-1}\left[\left(c^{T} b_{2}\right) v(k-1)+\left(c^{T} b_{1}\right) v(k-2)\right] \\
& +\left(c^{T} b\right)^{-1}[s(k)-\beta s(k-1)] .
\end{aligned}
$$

The compensation input $v(k)$ is defined as an estimate of $-d(k): v(k)=-\widehat{d}(k)=-d(k-1)$. From (36), the following perturbation compensation law can be obtained:

$$
\begin{aligned}
v(k)= & \left(c^{T} b\right)^{-1}\left[\left(c^{T} b_{2}\right) v(k-1)+\left(c^{T} b_{1}\right) v(k-2)\right] \\
& -\left(c^{T} b\right)^{-1}[s(k)-\beta s(k-1)] .
\end{aligned}
$$

Obviously, (37) is the decoupled perturbation compensator and the convergence rate of $|d(k)-v(k)|$ is only related to $\left(c^{T} b\right)^{-1}[s(k)-\beta s(k-1)]$. If the perturbation is constant or slowly varying, another adjustable parameter $\alpha(0<\alpha \leq 1)$ was added on the item that contains the switching function in (37) to make $|d(k)-v(k)|$ converges asymptotically to zero in a desired sliding function dynamics, and hence the following equations can be obtained:

$$
\begin{aligned}
v(k)= & \left(c^{T} b\right)^{-1}\left[\left(c^{T} b_{2}\right) v(k-1)+\left(c^{T} b_{1}\right) v(k-2)\right] \\
& -\alpha\left(c^{T} b\right)^{-1}[s(k)-\beta s(k-1)] .
\end{aligned}
$$

For a periodic disturbance with the known period $N$, the current disturbance can be actually obtained as a one-period shifted disturbance as

$$
\begin{aligned}
d(k-N) & \\
= & -\left(c^{T} b\right)^{-1}\left[\left(c^{T} b_{2}\right) v(k-N)+\left(c^{T} b_{1}\right) v(k-N-1)\right] \\
& +\alpha\left(c^{T} b\right)^{-1}[s(k-N+1)-\beta s(k-N)] .
\end{aligned}
$$

The compensation input $v(k)$ is defined as an estimate of $-\widehat{d}(k)=-d(k-N)$, thus,

$$
\begin{aligned}
v(k)= & \left(c^{T} b\right)^{-1}\left[\left(c^{T} b_{2}\right) v(k-N)+\left(c^{T} b_{1}\right) v(k-N-1)\right] \\
& -\alpha\left(c^{T} b\right)^{-1}[s(k-N+1)-\beta s(k-N)] .
\end{aligned}
$$

Obviously (40) is the decoupled compensator for periodic disturbance.

In theory, control signal can steer the errors from any finite value to the sliding surface in one step if the magnitude of the control signal can be arbitrarily large. In real control implementation, there is always a limitation $\left[u_{\min }, u_{\max }\right]$ for the actual control signal, whose amplitude must be within certain range. If the control input signal is too large, some components of the systems would be damaged. In order to avoid this, the range must be chosen so as to ensure the steering of the errors to the sliding surface in a finite number of steps instead of a single step. Similar to Bartolini et al. [14], in this study, the control input $u(k)$ is selected as follows:

$$
u(k)=\left\{\begin{array}{cc}
u(k) & \text { if }|u(k)|<u_{0} \\
\frac{u_{0} u(k)}{|u(k)|} & \text { if }|u(k)|>u_{0},
\end{array}\right.
$$

where $u_{0}$ is the actual control limit. This control law also forces the errors to reach the discrete-time sliding mode with a decreasing sequence $|d(k)-d(k-N)|$ [39]. 
Remark 4. If there exists no computation time delay, that is, if $\delta=0$, then the matrix $b_{2}$ becomes $b$, and the $b_{1}$ term disappears. When there exists the time delay, the forms of the control law and disturbance estimator had been changed due to $b_{1}$ and $b_{2}$, and so the complete disturbance rejection can also be achieved via the above (40).

\section{Application to a Hard Disk Drive}

4.1. Modeling the Plant. In hard disk drive (HDD) model, the rigid body model of the voice coil motor (VCM) and the resonance dynamics are the most important for servo control. The block diagram of the HDD servomechanism with DSMC is shown in Figure 1. The nominal model, that is, the rigid body dynamics of VCM, is formulated as a double integrator model as follows:

$$
H(s)=\frac{y(s)}{i_{c}(s)}=\frac{k_{v} k_{y}}{s^{2}},
$$

where $y$ (tracks) is the position of read/write head, $i_{c}$ is the VCM input current, $k_{y}$ is the position measurement gain, and $k_{v}$ is the acceleration constant.

There exist several resonance modes due to the flexibility of the actuator assembly. The transfer function for the resonance dynamics [34] can be expressed as follows:

$$
H_{d}(s)=\sum_{j=1}^{4} \frac{b_{2 j} \omega_{j} s+b_{2 j-1} \omega_{j}^{2}}{s^{2}+2 \xi \omega_{j} s+\omega_{j}^{2}} .
$$

During the controller design, system resonance dynamics was treated as model uncertainties and only double integrator model for the voice coil motor is considered, and as a result, the corresponding state space model of the system becomes

$$
\left\{\begin{array}{l}
\dot{y}(t) \\
\dot{h}(t)
\end{array}\right\}=\left[\begin{array}{ll}
0 & 1 \\
0 & 0
\end{array}\right]\left\{\begin{array}{l}
y(t) \\
h(t)
\end{array}\right\}+\left[\begin{array}{c}
0 \\
k_{h} k_{y}
\end{array}\right] u(t),
$$

where $y$ and hare the head displacement and velocity, respectively, and $u$ is the VCM coil current input. Let $F=\left[\begin{array}{ll}0 & 1 \\ 0 & 0\end{array}\right]$, $G=\left[\begin{array}{c}0 \\ k_{h} k_{y}\end{array}\right]$; if the sampling time is assumed to be $T_{s}$, using (8), $(A, b)$ and their the series expansion expression in [34], the matrix of discrete-time system $x(k+1)=A x(k)+b u(k)$ can be obtained as $\left(F^{2}=0\right.$ in this case $)$

$$
\begin{aligned}
A & =e^{F T_{s}}=I+F T_{s}+\frac{F^{2} T_{s}^{2}}{2 !}=\left[\begin{array}{ll}
1 & 0 \\
0 & 1
\end{array}\right]+\left[\begin{array}{ll}
0 & 1 \\
0 & 0
\end{array}\right] T_{s} \\
& =\left[\begin{array}{ll}
1 & 0 \\
0 & 1
\end{array}\right]+\left[\begin{array}{cc}
0 & T_{s} \\
0 & 0
\end{array}\right]=\left[\begin{array}{cc}
1 & T_{s} \\
0 & 1
\end{array}\right] \\
b & =\int_{0}^{T_{s}} e^{F \tau} d \tau G=\left[I T_{s}+F \frac{T_{s}^{2}}{2 !}\right] G \\
& =\left[\begin{array}{c}
\frac{k_{y} k_{h} T_{s}^{2}}{2} \\
k_{y} k_{h} T_{s}
\end{array}\right],
\end{aligned}
$$

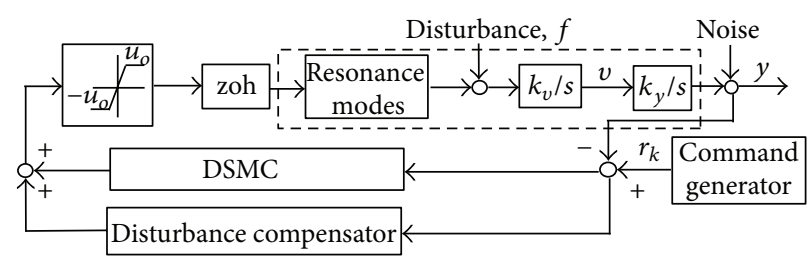

FIGURE 1: Block diagram of DSMC seeking control of an HDD.

and the discrete-time model of the nominal plant becomes

$$
\left\{\begin{array}{l}
y(k+1) \\
h(k+1)
\end{array}\right\}=\left[\begin{array}{cc}
1 & T_{s} \\
0 & 1
\end{array}\right]\left\{\begin{array}{l}
y(k) \\
h(k)
\end{array}\right\}+\left[\begin{array}{c}
\frac{k_{y} k_{h} T_{s}^{2}}{2} \\
k_{y} k_{h} T_{s}
\end{array}\right] u(k) .
$$

The measured frequency response function of the system is shown in Figure 2, and the system parameters can be identified as $k_{y}=63867.78 \mathrm{track} \cdot \mathrm{m}^{-1}, k_{h}=772.13 \mathrm{~m} \mathrm{~s}^{-2} \mathrm{~A}^{-1}$, $u_{\max }=0.25 \mathrm{~A}, T_{s}=1 / 12 \mathrm{e} 3 \mathrm{~s}$, and the spindle motor speed $4200 \mathrm{rpm}$. The periodic disturbance (RRO) $d(k)$ in HDD is assumed to be $d(k)=0.01 \sin 2 \pi\left(70 k T_{s}\right)+$ $0.005 \sin 2 \pi\left(140 k T_{s}\right)+0.003 \sin 2 \pi\left(210 k T_{s}\right)+$ $0.001 \sin 2 \pi\left(280 k T_{s}\right)+0.001 \sin 2 \pi\left(350 k T_{s}\right)$.

Let the computation time delay be $\delta$, using the expansion equation in [34] and following the above procedure, then the discrete-time model becomes

$$
\begin{aligned}
& \left\{\begin{array}{l}
y(k+1) \\
h(k+1)
\end{array}\right\}=\left[\begin{array}{cc}
1 & T_{s} \\
0 & 1
\end{array}\right]\left\{\begin{array}{l}
y(k) \\
h(k)
\end{array}\right\} \\
& +\left[\begin{array}{c}
\frac{k_{y} k_{h}\left(2 T_{s} \delta-\delta^{2}\right)}{2} \\
k_{y} k_{h} \delta
\end{array}\right] u(k-1) \\
& +\left[\begin{array}{c}
\frac{k_{y} k_{h}\left(T_{s}-\delta\right)^{2}}{2} \\
k_{y} k_{h}\left(T_{s}-\delta\right)
\end{array}\right] u(k) \text {. }
\end{aligned}
$$

4.2. Controller Design. The DSMC and the compensator for periodic disturbance are developed as (14) and (20). Based on the nominal plant model (47), that is, the double integrator model, the switching function is designed by defining $c^{T}=$ [10000 1]. The other parameters in the closed-loop model are chosen as $\beta=0.01$ and $\alpha=0.8$. The saturation for control effort is selected as \pm 0.25 . Simulation results are shown clearly in Figure 3. The periodic disturbance can be estimated accurately after three periods, as shown in Figure 3(a); the proposed method also reduces position error signal (PES) effectively due to the resultant periodic compensation control input, as shown in Figure 3(b). For parametric uncertainties and nonperiodic disturbances, the resonant modes of the head actuator are taken into account; that is, by adding the first fourth resonances to the SIMULINK model for nominal plant, the system with the proposed DSMC and compensator has been found to be just as stable and to exhibit the 


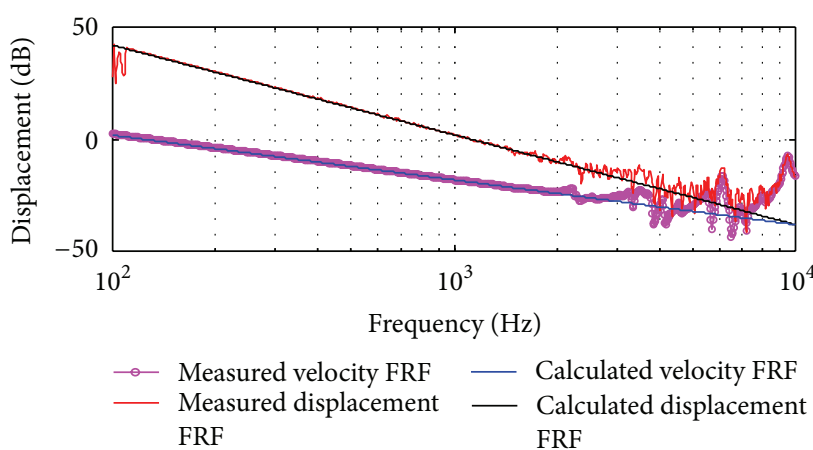

(a)

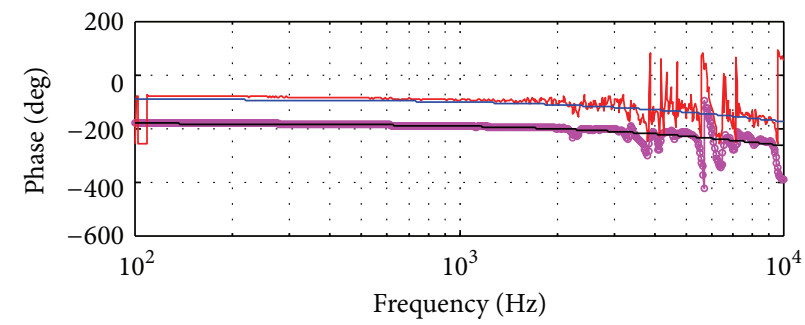

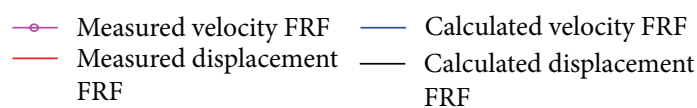

(b)

FIGURE 2: Measured versus the identified (double integrator model) FRFs.

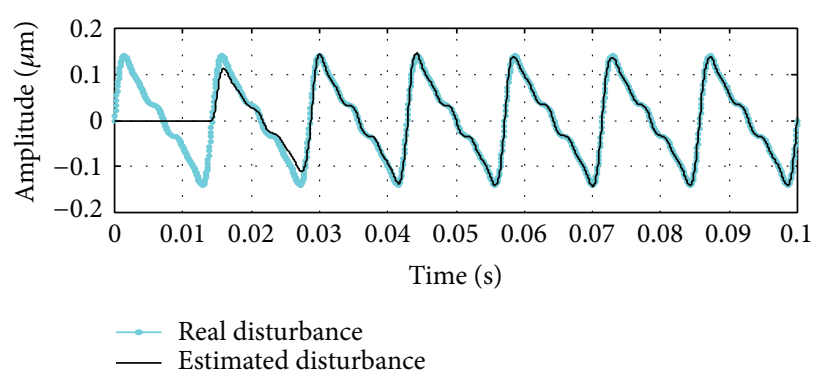

(a)

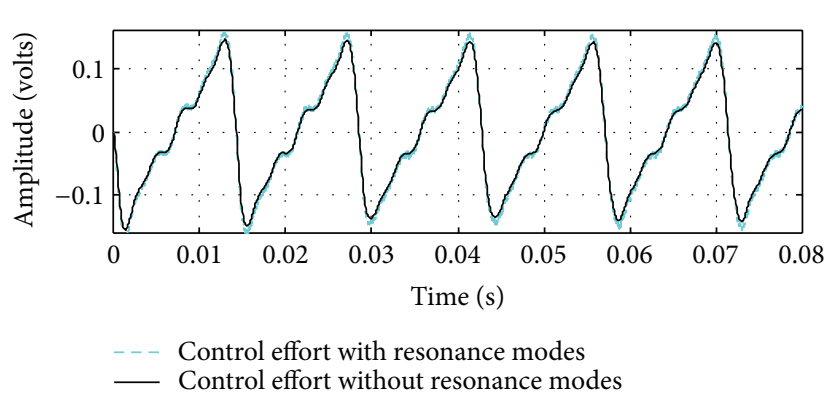

(c)

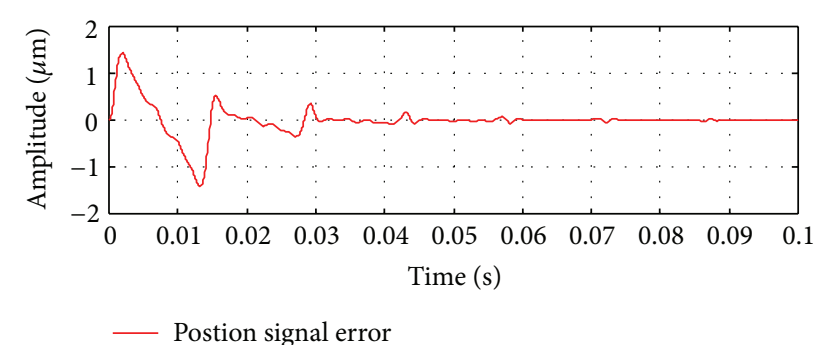

(b)

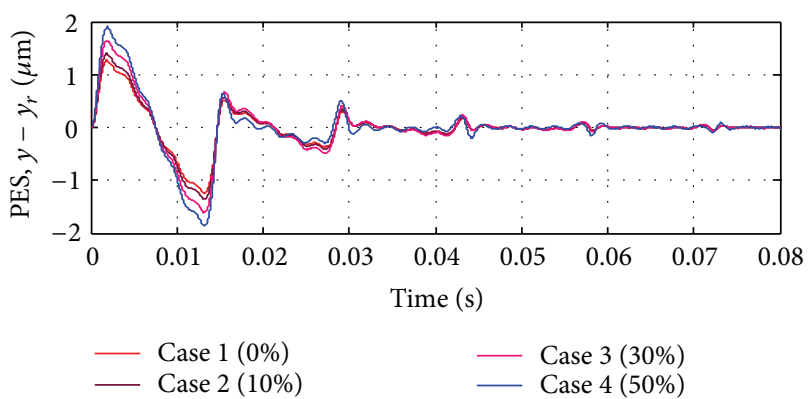

(d)

FIGURE 3: Simulation results of proposed DSMC for periodic disturbance compensation.

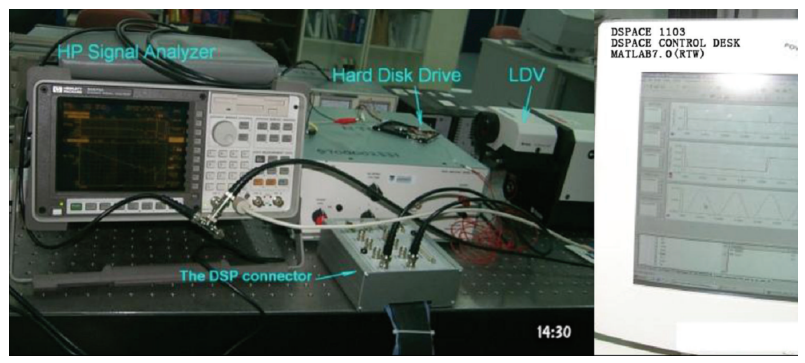

FIGURE 4: The experimental setup.

same performance. This demonstrates that the DSMC had effectively suppressed the uncertainties due to the resonance modes of the system with different control effort, as shown in Figure 3(c). To examine the robust stability to parameter variations, the plant model parameters are assumed to be estimated with errors from their nominal values, and here the resonant dynamics is still taken as part of model uncertainties. Figure 3(d) depicts the simulation results comparison using the same control parameters for the following fours cases: (1) no estimation error; (2) with $10 \%$ estimation error; (3) with $20 \%$ estimation error; (4) with $30 \%$ estimation error. Figure 3(d) clearly exhibits the robust performance of the designed controller.

\section{Experimental Results}

Figure 4 shows the experimental setup used for this investigation. An HP signal analyzer (HP 35670A) was used for modal testing. The control desk consists of the laser Doppler vibrometer (LDV), an HDD, and a PC installed with the DSpace 


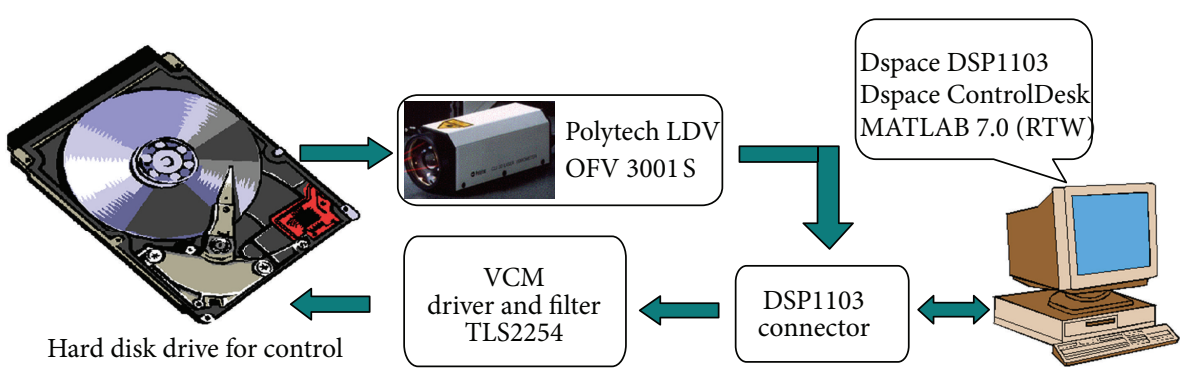

Figure 5: Control system configurations.

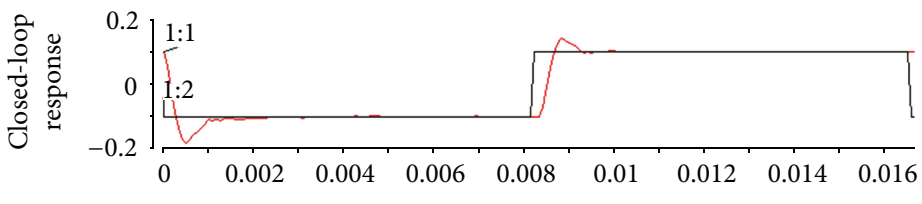

- 1:1 Closed-loop response (model root/polarity/out1)

- 1:2 Reference signal (model root/kref/out1)

(a)

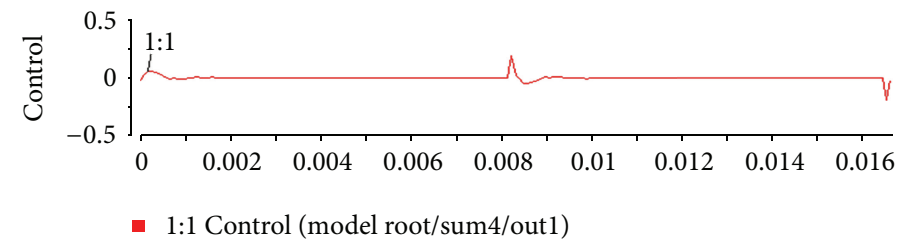

(b)

FIGURE 6: DSMC for track-to-track seeking of HDD without external disturbance.

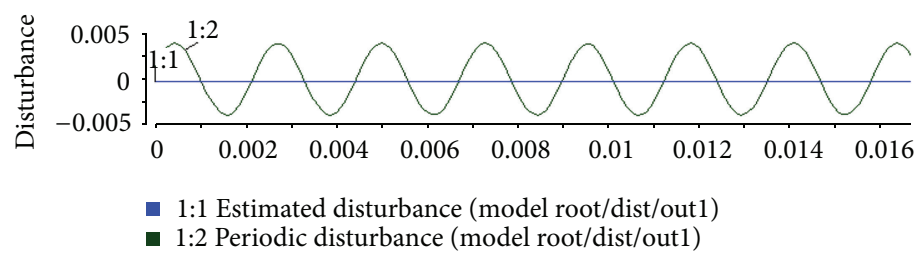

(a)

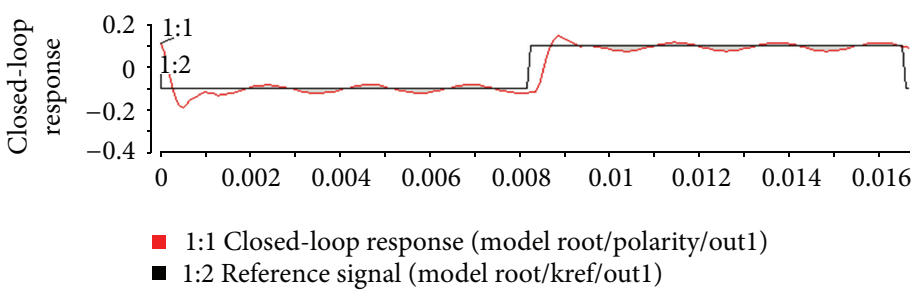

(b)

FIGURE 7: DSMC without disturbance compensator for track-to-track seeking of HDD with periodic disturbances (time delay $\delta=0$ ).

DSP system (DSPACE 1103) and the MATLAB software packages. The detailed configurations of the control system are shown in Figure 5. A sampling time of $T_{s}=83.33 \mu \mathrm{s}$ was used during the control implementation.

Experimental results for track-to-track seeking in different cases are shown in Figures 6-10. Figure 6 shows the closed-loop response of the system with the proposed DSMC after the parameters were adjusted to become $c^{T}=[95001]$, $\beta=0.16$, and $\alpha=0.85$ with $\pm 0.25 \mathrm{~A}$ as the saturation for control effort. The steady-state error of the closed-loop system is zero; that is, PES does not exist in track-to-track following by the DSMC when there does not exist external disturbance in the servo system. The settling time that occurs in the step response of time domain is just about $1 \mathrm{~ms}$ for one track. 


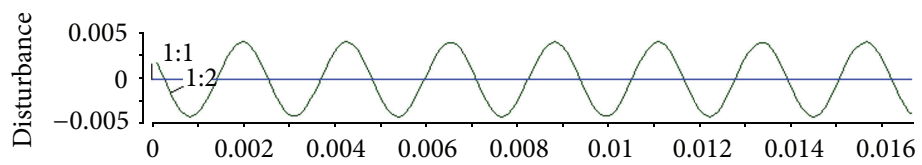

- 1:1 Estimated disturbance (model root/dist/out1)

- 1:2 Periodic disturbance (model root/dist/out1)

(a)

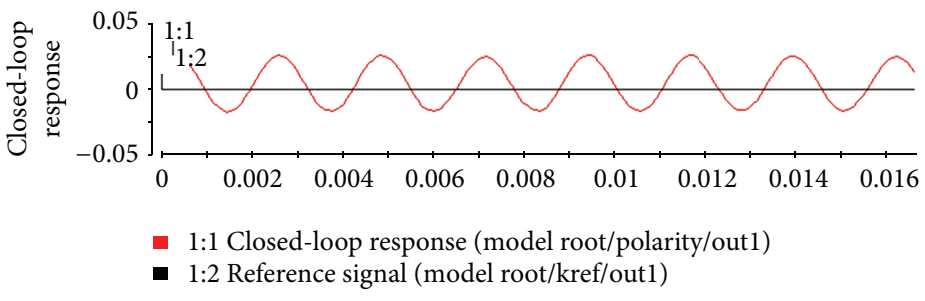

(b)

FIGURE 8: DSMC without disturbance compensator for track following of HDD with periodic disturbances (time delay $\delta=0$ ).

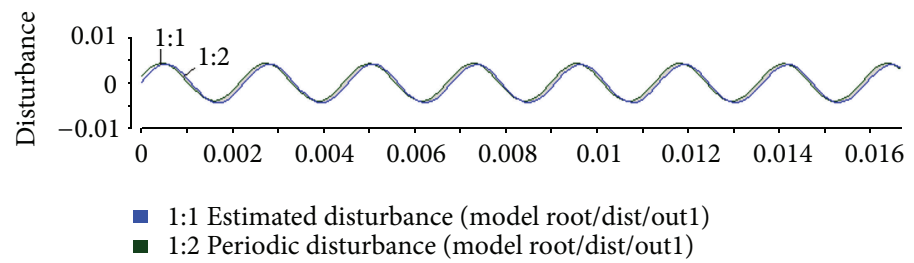

(a)

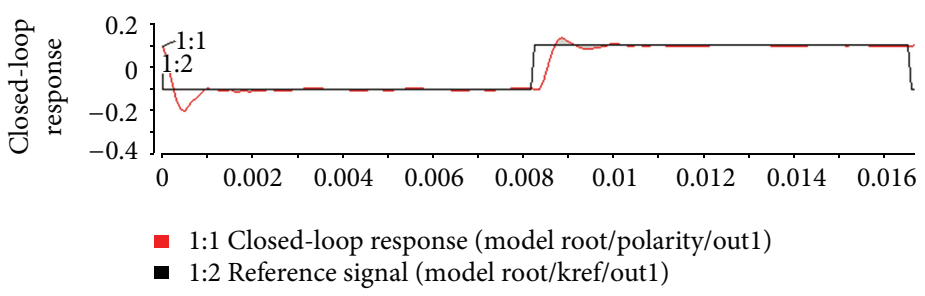

(b)

FIGURE 9: DSMC with disturbance compensator for track-to-track seeking of HDD with periodic disturbances (time delay $\delta=0)$.

The overshoot is also lower than half of the reference signal. When the external disturbances with frequency $420 \mathrm{~Hz}$ (period $N=420 \mathrm{~Hz}$ ) as shown in Figure 7 were added to the servo system, the PES of the closed-loop system with the proposed controller was increased significantly in the case where decoupled disturbance compensator was not incorporated into the control system. The PES of such closed-loop system for track following of HDD with RRO is very significant as shown in Figure 8. On the other hand, PES measurement of the closed-loop system with DSMC and decoupled periodic disturbance compensator for track-to-track seeking is shown in Figure 9. The amplitude of PES was greatly decreased from $25 \mathrm{mv}$ to $6.2 \mathrm{mv}$ after optimizing the parameters of the compensator, decreased more than $75 \%$ as compared with that of Figure 7. The PES of the closed-loop system for track following of HDD with RRO was significantly reduced when a disturbance compensator was incorporated, as shown in Figure 10. Compared with Figure 8, it can be seen clearly that the PES has been reduced by more than $75 \%$. As shown in Figure 10, however, the disturbance could not be compensated completely probably due to the computation time delay as will be shown next.

Experimental PES measurement of DSMC with disturbance compensator that considers the computation time delay is shown in Figures 11 and 12.

After the computation time delay was adjusted to become $\delta=30.0 \mu \mathrm{s}$, responses of DSMC with disturbances compensation for track-to-track seeking were measured and are shown in Figure 11. Obviously, from Figure 11, periodic disturbance has almost been compensated completely, and virtually there is no difference between the reference and the steady response. The effectiveness of DSMC and disturbance compensator with computation time delay can be also seen from the experimental results obtained from track following 


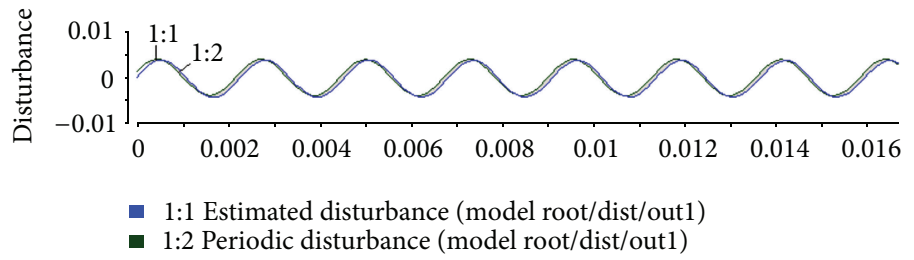

(a)

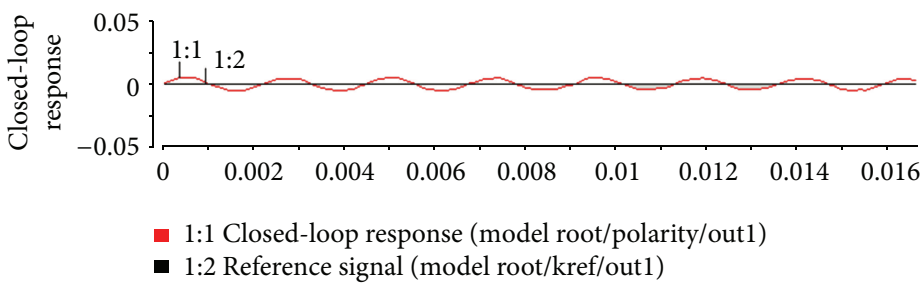

(b)

FIGURE 10: DSMC with disturbance compensator for track following of HDD with periodic disturbances (time delay $\delta=0$ ).

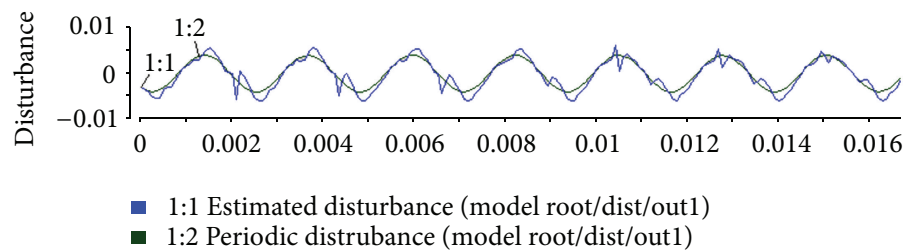

(a)

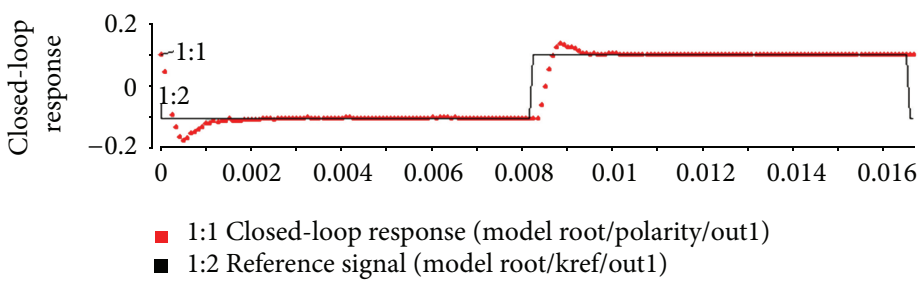

(b)

FIGURE 11: DSMC with disturbance compensator for track-to-track seeking of HDD with periodic disturbances (time delay $\delta=30.0 \mu$ ).

control, as shown in Figure 12. From which, it can be seen clearly that PES has been suppressed considerably.

\section{Conclusions}

A method of achieving robustness for perturbation rejection in applying discrete-time sliding mode control has been presented. The developed DMSC with DPC control method, which considers computation time delay, achieves robust tracking in the presence of unknown disturbances, which include external disturbances as well as parameter uncertainties. The presented method decouples the disturbance estimation dynamics from the closed-loop sliding mode dynamics. An additional parameter has been introduced to allow the asymptotic convergence in a desired sliding dynamics in both tracking and regulation problems. The satisfied simulation results as well as experimental results based on the servomechanism of a modern $2.5^{\prime \prime}$ HDD have been achieved to demonstrate the practicality of the proposed method. The simulation results show the satisfactory feature of the complete rejection of the periodic component. In the experimental implementation of the control based on the DSP1102, the performances of the closed-loop system using DSMC and the perturbation compensator control were improved greatly when computation time delay was considered. This demonstrates the robustness of the sliding mode dynamics to parameter uncertainties and exogenous disturbances containing periodic components and such robustness is always guaranteed for developed control scheme.

\section{Acknowledgments}

This work was supported by the Natural Science Foundation of China (51075377, 41176076, 51121002, and 51175486), 


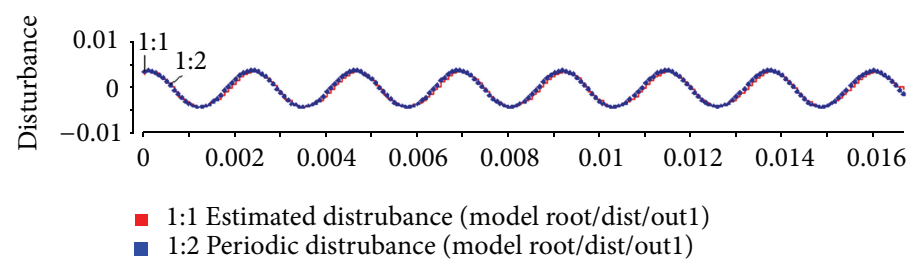

(a)

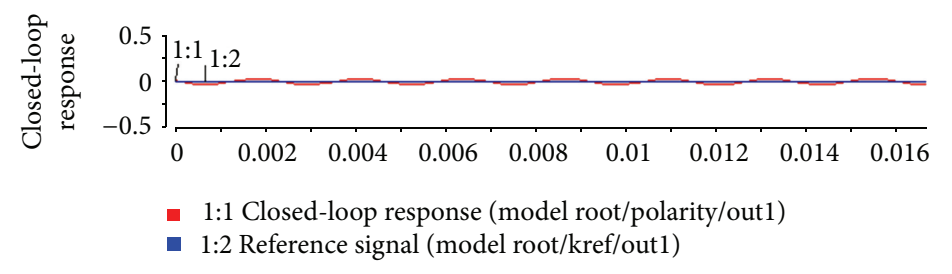

(b)

FIGURE 12: DSMC with disturbance compensator for track following of HDD with periodic disturbances (time delay $\delta=30.0 \mu \mathrm{s}$ ).

Natural Science Foundation of Zhejiang province (R1100015, Y7100512), and Open Foundation of State Key Laboratory of Fluid Power and Mechatronic Systems in Zhejiang University (GZKF-201017).

\section{References}

[1] U. Itkis, Control Systems of Variable Structure, Wiley, New York, NY, USA, 1976.

[2] V. I. Utkin, "Variable structure systems with sliding modes," Institute of Electrical and Electronics Engineers. Transactions on Automatic Control, vol. 22, no. 2, pp. 212-222, 1977.

[3] S. Hui and S. H. Żak, "On discrete-time variable structure sliding mode control," Systems \& Control Letters, vol. 38, no. 4-5, pp. 283-288, 1999.

[4] K. Kalsi, J. Lian, S. Hui, and S. H. Żak, "Sliding-mode observers for systems with unknown inputs: a high-gain approach," Automatica, vol. 46, no. 2, pp. 347-353, 2010.

[5] J. X. Xu, T. H. Lee, and Y. J. Pan, "On the sliding mode control for DC servo mechanisms in the presence of unmodeled dynamics," Mechatronics, vol. 13, no. 7, pp. 755-770, 2003.

[6] W. C. Wu and T. S. Liu, "Sliding mode based learning control for track-following in hard disk drives," Mechatronics, vol. 14, no. 8, pp. 861-876, 2004.

[7] J. P. F. Garcia, L. M. C. F. Garcia, G. C. Apolinário, and F. B. Rodrigues, "Sliding mode for detection and accommodation of computation time delay fault," Mathematics and Computers in Simulation, vol. 80, no. 2, pp. 449-465, 2009.

[8] J. H. Kim, S. H. Oh, D. I. Cho, and J. K. Hedrick, "Robust discrete-time variable structure control methods," Transactions of the ASME Journal of Dynamic Systems, Measurement and Control, vol. 122, no. 4, pp. 766-775, 2000.

[9] K. Furuta, "Sliding mode control of a discrete system," Systems \& Control Letters, vol. 14, no. 2, pp. 145-152, 1990.

[10] S. Z. Sarpturk, Y. Istefanopulos, and O. Kaynak, "On the stability of discrete-time sliding mode control systems," IEEE Transactions on Automatic Control, vol. 32, pp. 930-932, 1987.
[11] U. Kotta, "Comments on 'On the stability of discrete-time sliding mode control systems," IEEE Transactions on Automatic Control, vol. 34, no. 9, pp. 1021-1022, 1989.

[12] J. Y. Hung, W. Gao, and J. C. Hung, "Variable structure control: a survey," IEEE Transactions on Industrial Electronics, vol. 40, pp. 2-22, 1993.

[13] W. Gao, Y. Wang, and A. Homaifa, "Discrete-time variable structure control systems," IEEE Transactions on Industrial Electronics, vol. 42, no. 2, pp. 117-122, 1995.

[14] G. Bartolini, A. Ferrara, and V. I. Utkin, "Adaptive sliding mode control in discrete-time systems," Automatica, vol. 31, no. 5, pp. 769-773, 1995.

[15] A. Tesfaye and M. Tomizuka, "Robust control of discretized continuous systems using the theory of sliding modes," International Journal of Control, vol. 62, no. 1, pp. 209-226, 1995.

[16] W.-C. Su, S. V. Drakunov, and Ü. Özgüner, "Implementation of variable structure control for sampled-data systems," in Robust Control via Variable Structure and Lyapunov Technique, F. Garofalo and L. Glielmo, Eds., vol. 217 of Lecture Notes in Control and Information Sciences, pp. 87-106, Springer, Berlin, Germany, 1996.

[17] A. J. Koshkouei and A. S. I. Zinober, "Discrete-time sliding mode control design," in Proceedings of the 13th IFAC world congress, pp. 481-486, 1996.

[18] M. L. Corradini and G. Orlando, "Variable structure control for uncertain sampled data systems," in Proceedings of the IEEE International Workshop on Variable Structure Systems (VSS '96), pp. 117-121, December 1996.

[19] W.-C. Su, S. V. Drakunov, and Ü. Özgüner, "An O(T $\left.{ }^{2}\right)$ boundary layer in sliding mode for sampled-data systems," IEEE Transactions on Automatic Control, vol. 45, no. 3, pp. 482-485, 2000.

[20] Y. Eun, J. H. Kim, K. Kim, and D. I. Cho, "Discrete-time variable structure controller with a decoupled disturbance compensator and its application to a CNC servomechanism," IEEE Transactions on Control Systems Technology, vol. 7, no. 4, pp. 414-423, 1999.

[21] J. Kim and D. Cho, "Discrete-time variable structure control using recursive switching function," in Proceedings of the American Control Conference, pp. 1113-1117, 2000. 
[22] S. M. Lee and B. H. Lee, "A discrete-time sliding mode controller and observer with computation time delay," Control Engineering Practice, vol. 7, no. 8, pp. 943-955, 1999.

[23] R. Kondo and K. Furuta, "Sampled-data optimal control of continuous systems for quadratic criterion function taking account of delayed control action," International Journal of Control, vol. 41, no. 4, pp. 1051-1060, 1985.

[24] C. Ha and U. L. Ly, "Sampled-data system with computation time delay: optimal W-synthesis method," Journal of Guidance, Control, and Dynamics, vol. 19, no. 3, pp. 584-591, 1996.

[25] E. A. Misawa, "Observer-based discrete-time sliding mode control with computational time delay: the linear case," in Proceedings of theAmerican Control Conference, pp. 1323-1327, June 1995.

[26] M. Tomizuka, T. C. Tsao, and K. K. Chew, "Analysis and synthesis of discrete-time repetitive controllers," Transactions of the ASME Journal of Dynamic Systems, Measurement and Control, vol. 111, no. 3, pp. 353-358, 1989.

[27] P. Ignaciuk and A. Bartoszewicz, "Discrete-time sliding-mode congestion control in multisource communication networks with time-varying delay," IEEE Transactions on Control Systems Technology, vol. 19, no. 4, pp. 852-867, 2011.

[28] X.-G. Yan, S. K. Spurgeon, and C. Edwards, "Sliding mode control for time-varying delayed systems based on a reducedorder observer," Automatica, vol. 46, no. 8, pp. 1354-1362, 2010.

[29] Q. Hu, C. Du, L. Xie, and Y. Wang, "Discrete-time sliding mode control with time-varying surface for hard disk drives," IEEE Transactions on Control Systems Technology, vol. 17, no. 1, pp. 175-183, 2009.

[30] E. Azadi Yazdi, M. Sepasi, F. Sassani, and R. Nagamune, "Automated multiple robust track-following control system design in hard disk drives," IEEE Transactions on Control Systems Technology, vol. 19, no. 4, pp. 920-928, 2011.

[31] J. Nie and R. Horowitz, "Control design of hard disk drive concentric self-servo track writing via $\mathrm{H}_{2}$ and $H_{\infty}$ synthesis," IEEE Transactions on Magnetics, vol. 47, no. 7, pp. 1951-1957, 2011.

[32] T. Semba, M. T. White, and F. Y. Huang, "Adaptive cancellation of self-induced vibration," IEEE Transactions on Magnetics, vol. 47, no. 7, pp. 1958-1963, 2011.

[33] J. T. Fei, T. H. Li, F. Wang, and W. R. Juan, "A novel sliding mode control technique for indirect current controlled active power filter," Mathematical Problems in Engineering, vol. 2012, Article ID 549782, 18 pages, 2012.

[34] G. F. Franklin, J. D. Powell, and M. L. Workman, Digital Control of Dynamic Systems, Addison-Wesley, Reading, Mass, USA, 3rd edition, 1998.

[35] A. J. Koshkouei and A. S. I. Zinober, "Sliding mode state observers for SISO linear discrete-time systems," in Proceedings of the UKACC International Conference on Control, vol. 96, pp. 837-842, September 1996.

[36] K. D. Young, V. I. Utkin, and Ü. Özgüner, "Control engineer's guide to sliding mode control," in Proceedings of the 1996 International Workshop on Variable Structure Systems (VSS '96), pp. 1-14, December 1996.

[37] C. Y. Chan, "Robust discrete-time sliding mode controller," Systems \& Control Letters, vol. 23, no. 5, pp. 371-374, 1994.

[38] A. J. Koshkouei and A. S. I. Zinober, "Sliding lattice design for discrete-time linear multivariable systems," in Proceedings of the 35th IEEE Conference on Decision and Control, pp. 1497-1502, December 1996.
[39] M. X. Sun and Y. Y. Wang, "Periodic disturbance compensation in discrete-time sliding mode control," in Proceedings of the International Conference on Control and Automation (ICCA '02), Xiamen, China, 2002. 


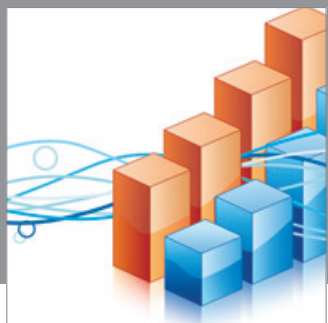

Advances in

Operations Research

mansans

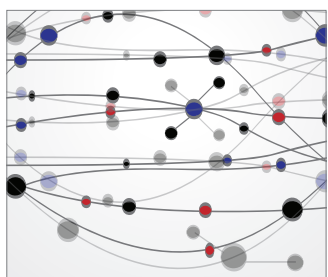

The Scientific World Journal
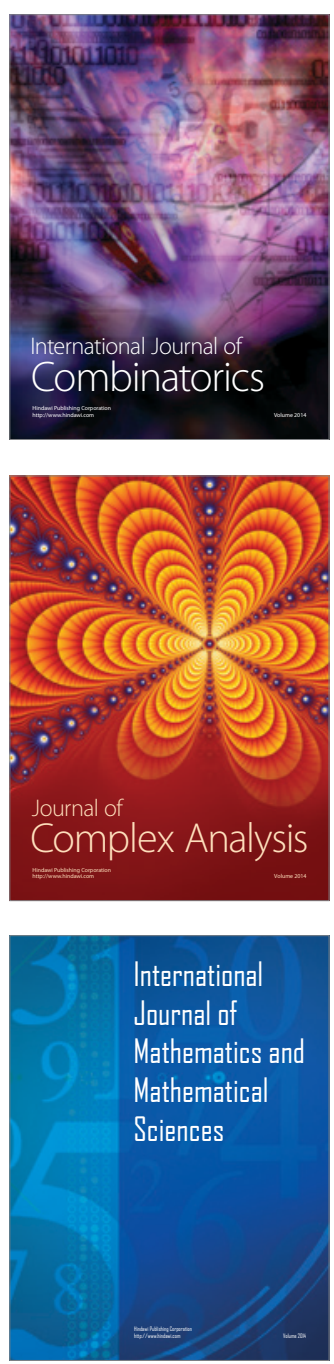
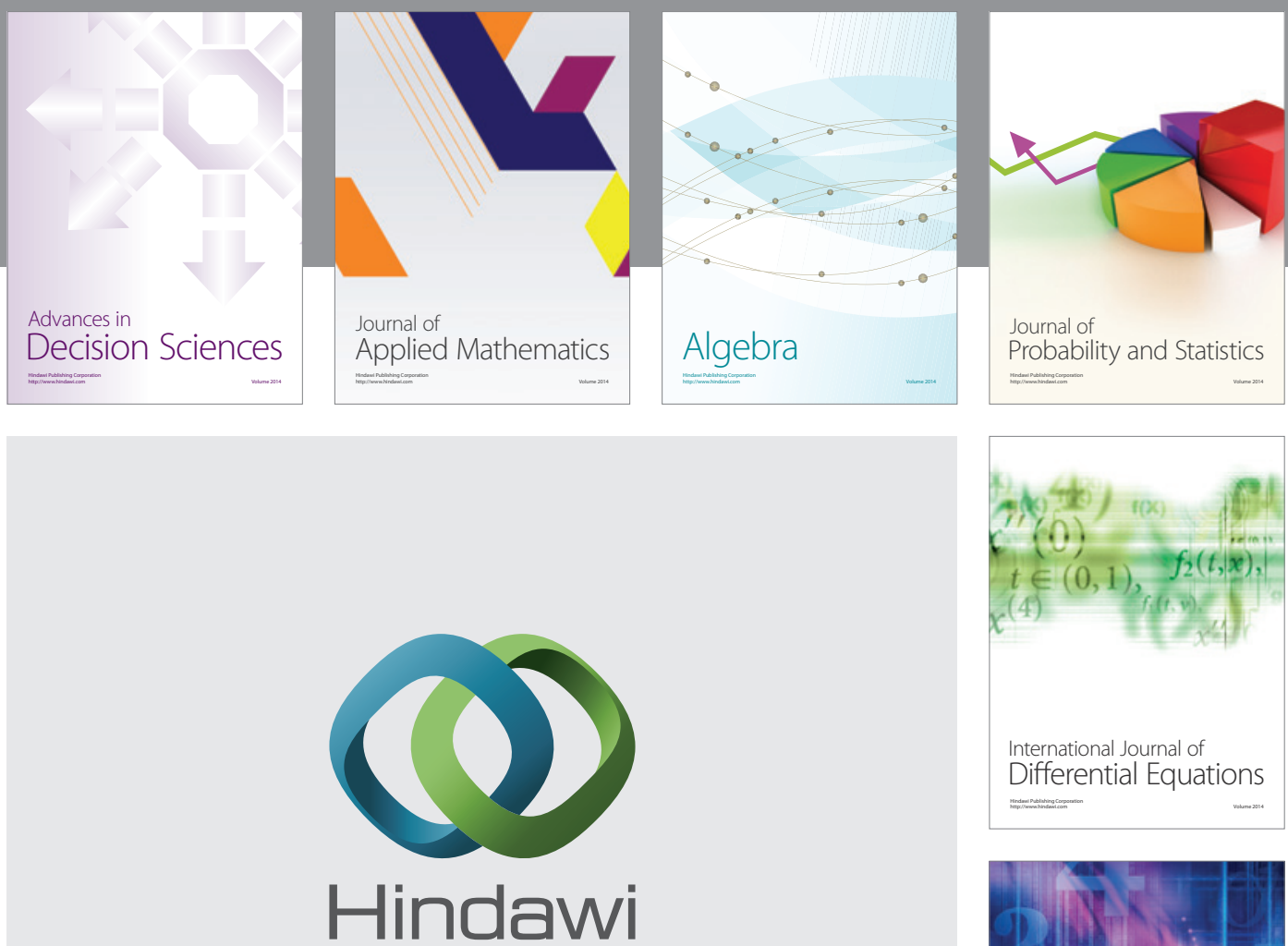

Submit your manuscripts at http://www.hindawi.com
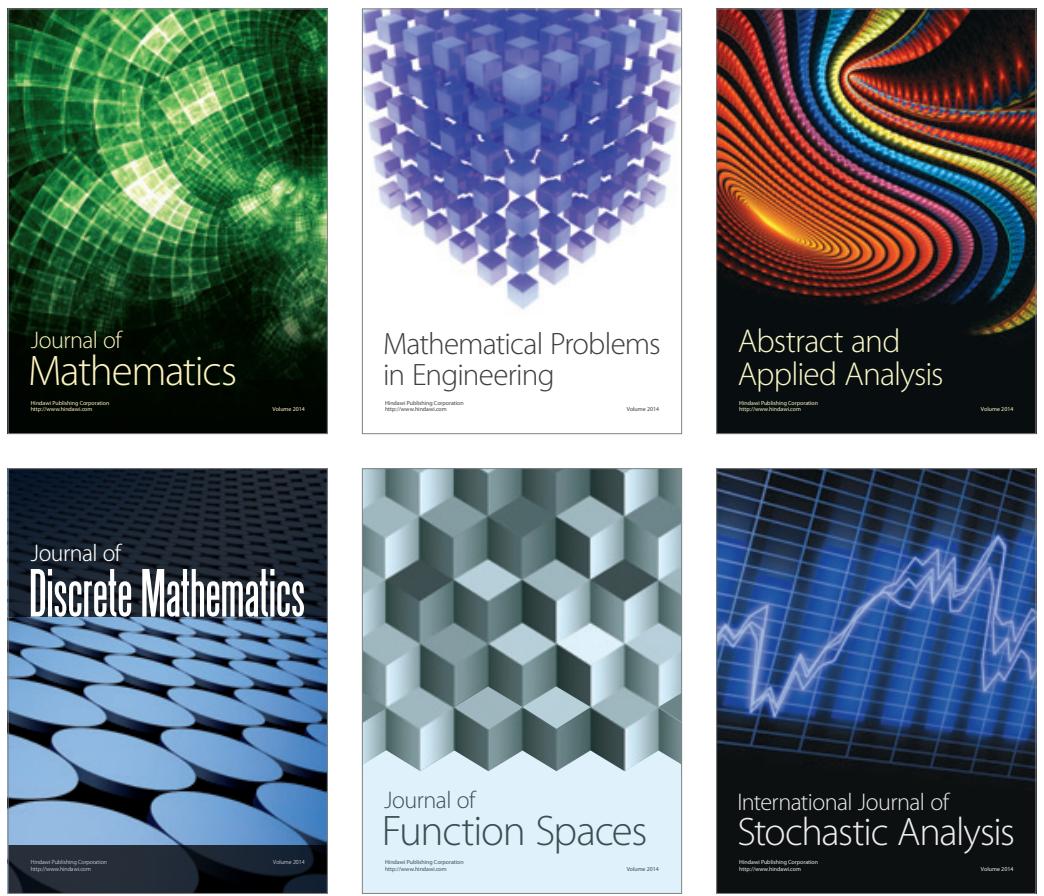

Journal of

Function Spaces

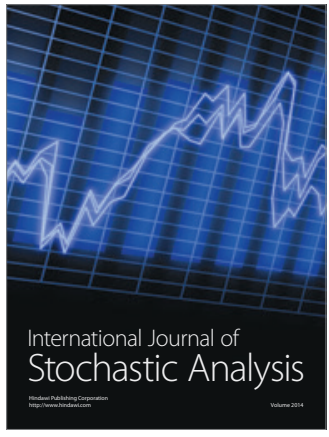

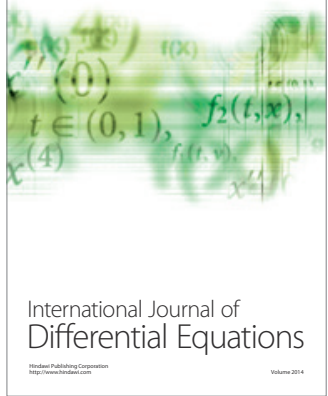
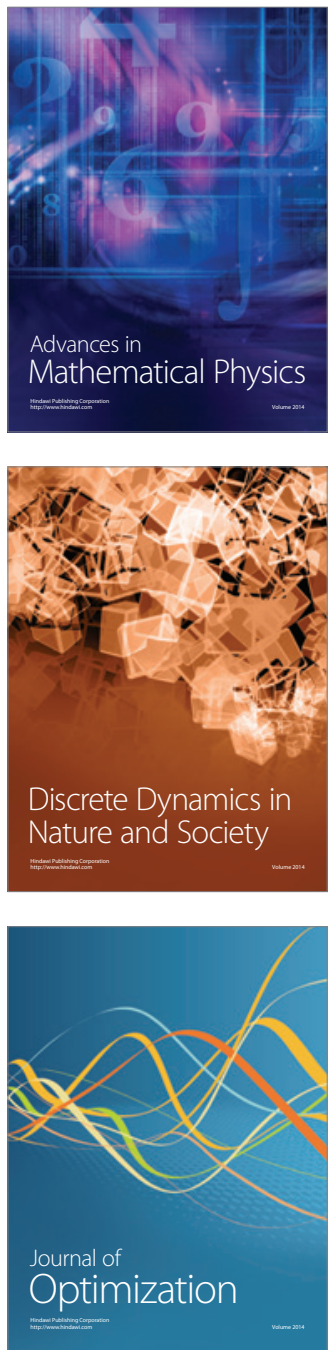